\title{
Avistamiento de ballenas en el Perú: estado actual y recomendaciones para su buen control
}

Whale watching in

Peru: Current status and recommendations for its proper control

Ana Belén Reyes Weydert ${ }^{1 *} \odot$

1 Carrera de Biología Marina, Universidad Cientifica del Sur. Lima, Perú.

\section{SOUTH} SUSTAINABILITY

Citar como: Reyes Weydert, A. B.

(2021). «Avistamiento de ballenas en el Perú: estado actual y recomendaciones para su buen control». South

Sustainability, 2(2), e043.

DOI: https://doi.org/10.21142/SS-

0202-2021-pb005

Artículo recibido: 13/11/2021

Arbitrado por pares

Artículo aceptado: 31/12/2021

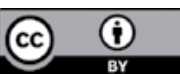

(c) La autora, 2021. Publicado por la Universidad Científica del Sur (Lima, Perú)

${ }^{*} E$-mail de correspondencia: reywey95@gmail.com

\section{RESUMEN}

La actividad de avistamiento de ballenas jorobadas en el norte del Perú, durante su temporada de reproducción y crianza, viene experimentando un crecimiento significativo en el sector recreativo. Esta actividad es regulada a través de la Resolución Ministerial 451-2019-PRODUCE, la cual solo menciona distancias mínimas de acercamiento a los cetáceos. Se proponen ocho sugerencias fundamentales para la mejora de esta actividad: un adecuado posicionamiento, distancia y cantidad máxima de embarcaciones, regulación del tiempo máximo de avistamiento, disminución de velocidad en la zona de precaución, alejamiento en dirección opuesta al individuo o grupo e indicaciones en caso de avistar un individuo enmallado. Estas sugerencias favorecerán la conservación de la especie y que la actividad sea sostenible en el tiempo en términos económicos, educativos y turísticos.

Palabras clave: avistamiento de ballenas, ballena jorobada, manejo sostenible, turismo

ABSTRACT

Humpback whale watching activities in northern Peru have been experiencing significant growth in the recreational sector. Such activities are regulated through RM - No 451-2019-PRODUCE, which only mentions minimum distances when approaching cetaceans. In this paper, eight fundamental suggestions are proposed for improvements in whale watching activities: adequate positioning, distance and maximum number of vessels, regulating of the maximum time permitted for whale watching, reduction in speed when entering the precautionary zone, withdrawal in the opposite direction to the individual or group, and indications in the event that an entangled whale is sighted. These suggestions will benefit the conservation of the species and the sustainability of whale watching activities over time, in economic, educational and tourism terms.

Keywords: humpback whale, sustainable management, tourism, whale watching 


\section{Introducción}

El avistamiento de ballenas es una de las actividades de ecoturismo de más rápido crecimiento en el mundo (Hoyt y Parsons, 2014; O'Connor et al., 2009). En el norte del Perú (Piura y Tumbes), esta actividad ocurre durante la temporada de reproducción y crianza del Stock $G$ de «ballenas jorobadas» (Megaptera novaeangliae), ya que esta zona presenta temperaturas cálidas (Castro et al., 2015; Guidino et al., 2014; Pacheco et al., 2011; Swartzman et al., 2008; Rasmussen et al., 2007; Stevick et al., 2004; Mackintosh, 1965).

La sola presencia de estos individuos origina un flujo importante de turismo por los despliegues superficiales que realizan (Félix, 2004). Debido a esto, el Ministerio de la Producción promulgó en 2019 la Resolución Ministerial 451-2019-PRODUCE, la cual determina las distancias mínimas de aproximación de cetáceos. No obstante, dicho documento no menciona pautas adicionales que se deberían considerar para su buen manejo, como las relacionadas con la velocidad de aproximación, el tiempo de duración con los diferentes grupos de cetáceos y la cantidad de embarcaciones. Sin un control y gestión adecuados, esta actividad puede generar un impacto negativo en el comportamiento y la reproducción de las ballenas jorobadas (Christiansen y Lusseau, 2013; Di Clemente et al., 2018).

En un escenario donde se ha evidenciado un incremento turístico a lo largo de los años (Guidino et al., 2020), nace la necesidad de describir la problemática actual del avistamiento de ballenas en el norte del Perú, así como también de proveer de pautas y recomendaciones basadas en la bibliografía disponible. El objetivo de este documento es brindar una herramienta que permita prevenir los impactos potenciales sobre las ballenas jorobadas, ante el comportamiento de los operadores turísticos de la zona.

\section{Enfoque y discusión}

Si bien el crecimiento de las actividades de avistamiento de ballenas como una alternativa turística ha sido beneficioso en términos económicos, el control de esta actividad sigue siendo un desafío para la mayoría de países que la realizan. Esto ocurre por un monitoreo gubernamental deficiente y la consecuente falta de regulaciones, la competencia entre los operadores turísticos, su falta de autoorganización y otras amenazas antropogénicas (Pacheco et al., 2021). Por este motivo, diferentes autores que han hecho estudios previos en la zona norte del Perú mencionan que, a medida que ocurre este crecimiento, la actividad puede generar efectos negativos en la población de ballenas jorobadas, y sugieren que se desarrolle una serie de recomendaciones y normas con apoyo de entes reguladores para lograr un correcto manejo de la actividad (Kessler y Harcourt, 2013; Chalcobsky et al., 2017; Garcia Cegarra et al., 2018; Gleason y Parsons, 2019; Pacheco et al., 2019; Schuler et al., 2019; Burnham et al., 2021).

La presencia de embarcaciones de avistamiento de ballenas induce a respuestas a corto plazo en los diferentes grupos de ballenas jorobadas (madre-cría, grupo competencia, solitarios, madre-cría-escolta). Las respuestas de cambio de comportamiento más observadas son el aumento de la velocidad de nado, el cambio en la dirección de la trayectoria, el incremento de la frecuencia de respiración, el aumento en el tiempo de inmersión, y la variación de los despliegues que realizan en superficie (por ejemplo, los saltos), de estar en actividad a buceos profundos (Garcia Cegarra et al., 2018). Generalmente, los individuos o grupos sin crías presentan cambios de velocidad de nado según la presencia de embarcaciones, el número de embarcaciones y el tiempo de permanencia con ellas. Cabe resaltar que la presencia de una, dos o tres embarcaciones no lleva a las jorobadas a cambios significativos de comportamiento. Esto solo ocurre cuando este número se incrementa a más de tres y el tiempo de permanencia con ellas es elevado, ya que los niveles de ruido provocados por los motores podrían inducir a cambios en su comportamiento (Villagra et al., 2021; Sprogis et al., 2020).

El grupo más vulnerable es el de madre-cría y es el más susceptible a la presencia de embarcaciones. En zonas de reproducción, las jorobadas dependen fisiológicamente de

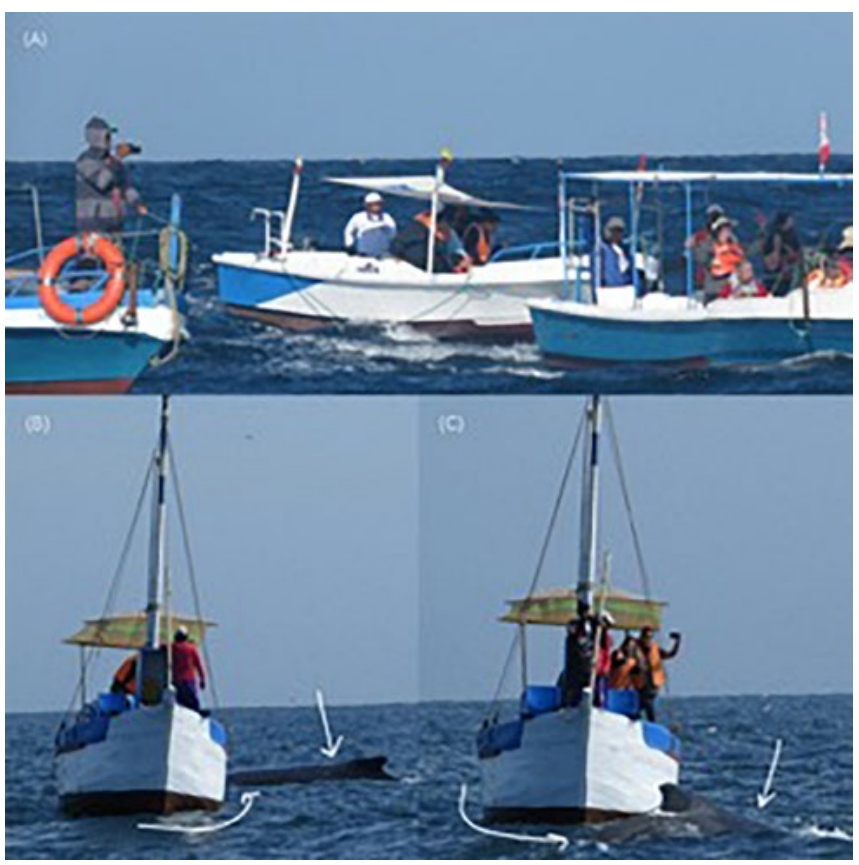

Figura 1. Material fotográfico obtenido en los tours de avistamiento de ballenas (julio-agosto de 2016-2018; Los Órganos, Piura). (A) Aglomeración de embarcaciones de avistamiento de ballenas sin formar una línea paralela (línea de tren) con el individuo avistado. $(B, C)$ Persecución de ballena jorobada en círculos, que causan una perturbación en su comportamiento y dirección de nado. Las flechas indican la ubicación de las ballenas. 
su reserva de grasa, y su energía es utilizada principalmente para dar a luz y transferir energía (como leche y grasa) a su cría, además de actividades de interacción social y actividades reproductivas en general. Por lo tanto, cuando una embarcación perturba al grupo madre-cría, se pueden presentar movimientos de salida por parte de la madre, buceos prolongados, cambios de dirección y aumento de velocidad de nado, lo que reduce el tiempo de lactancia de la cría y afecta su desarrollo corporal, así como el comportamiento social aprendido (Garcia Cegarra et al., 2018). Estas reacciones se manifiestan por una estrategia energéticamente eficiente que las ballenas jorobadas realizan para evitar una amenaza percibida, sobre todo en zonas de reproducción para proteger a sus crías (Schuler et al., 2019; Senigaglia et al., 2016).

Actividades que influencian en los cambios de comportamiento de las ballenas jorobadas han sido evidenciadas en el norte del Perú (figura 1).

Debido a que el Stock $G$ de ballenas jorobadas llega a la zona norte del Perú para reproducirse, parir y criar a sus ballenatos, se proponen las siguientes pautas y regulaciones (figura 2). Estas han sido adaptadas de experiencias previas de otros países y se basan en las recomendaciones listadas por Carlson (2010) y la International Whailing Comission, incluyendo la reglamentación descrita en la Resolución Ministerial 451-2019-PRODUCE (Material suplementario T1):

- Zona de precaución: es el espacio territorial donde las embarcaciones deberían bajar la velocidad de acercamiento. También sirve como zona de espera de embarcaciones. Empieza desde los 400 metros de distancia entre embarcación y ballena.

- Comportamiento de aproximación, alejamiento y seguimiento: la velocidad de acercamiento a las ballenas debe ser gradual. Se debe evitar acelerar dentro a la zona de los 400 metros, no seguir a las ballenas y partir en dirección opuesta al grupo de ballenas avistadas. Al alejarse del grupo, salir en dirección opuesta sin acelerar, hasta estar a los 400 metros de distancia.

- Limitaciones de tiempo: se recomienda no sobrepasar el tiempo de avistamiento a más de 25 minutos por individuo, especialmente si es madre con cría, ya que es el grupo más vulnerable.

- Posición de las embarcaciones: las embarcaciones deben posicionarse en paralelo a la ballena, formando una fila en tren entre las embarcaciones y respetando el turno de llegada. Una vez cumplido el tiempo, la primera debe retirarse en dirección opuesta al individuo avistado.

- Cantidad máxima de embarcaciones: evitar sobrepasar el número de tres embarcaciones por grupo o individuo.

- Distancia mínima de acercamiento: la distancia mínima más común de acercamiento a las ballenas es de 100 metros para los adultos. Se recomienda una distancia de 200 metros para los grupos con crías. En caso de que las ballenas se acerquen al bote, el motor debe permanecer en neutro hasta que estas se alejen voluntariamente.

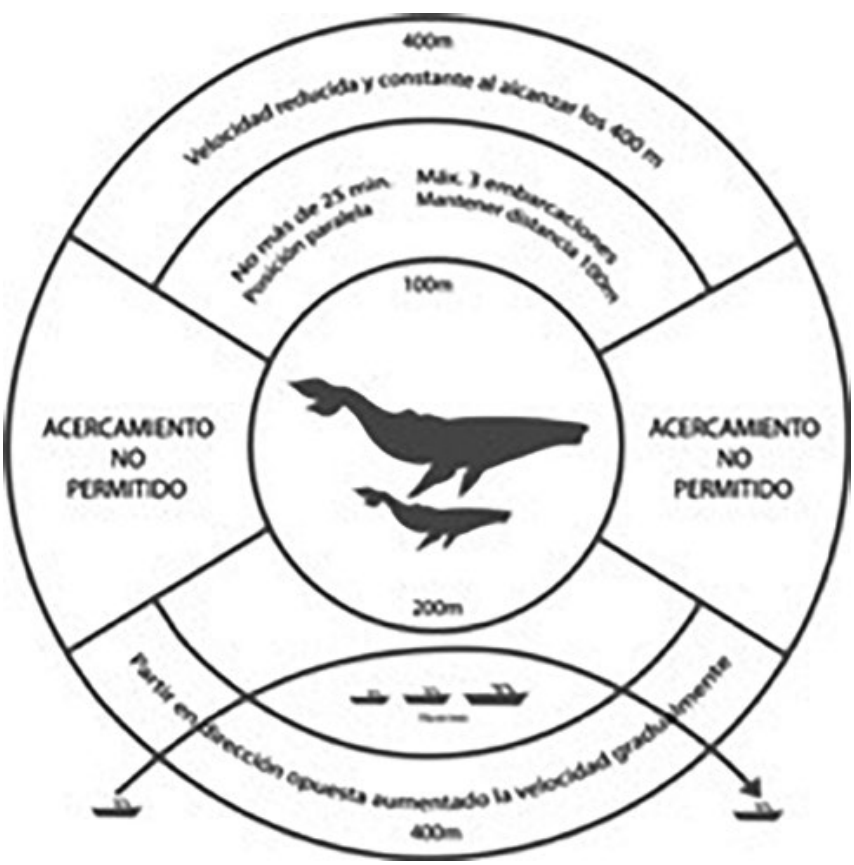

Figura 2. Infografía que reúne las pautas para la correcta actividad de avistamiento de ballenas de acuerdo con lo mencionado en la Resolución Ministerial 451-2019-PRODUCE y Carlson (2012).

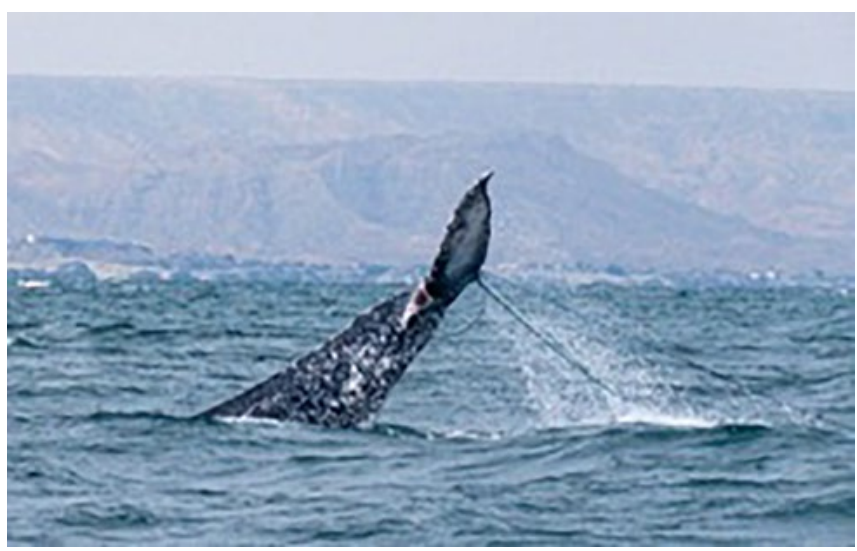

Figura 3. Material fotográfico de ballena jorobada atrapada en red de pesca obtenido en tour de avistamiento de ballenas (agosto de 2017; Los Órganos, Piura).

- Signos de perturbación: en caso se evidencien cambios de velocidad o dirección, se requiere alejarse del grupo de ballenas para evitar más variaciones en su comportamiento.

- Enmallamiento o daños físicos visibles: en caso de avistar un individuo enmallado o con algún daño físico, mantener una distancia segura y hacer el menor ruido posible. No intentar liberar al individuo, ya que puede atentar contra su seguridad. Comunicarse de inmediato con capitanía de puerto o el Instituto del Mar del Perú (Imarpe), para que tomen las acciones correspondientes.

En este contexto, se ha demostrado que el turismo en espacios naturales brinda oportunidades educativas de conservación y oportunidades económicas que pueden 
ser aprovechadas por los guías turísticos durante la realización de esta actividad (Guidino et al., 2020). Pero de acuerdo con la bibliografía revisada, existe una perturbación en el comportamiento de la población objetivo, cuya supervivencia se puede ver afectada no solo por esta actividad, sino también por redes de pesca o cabos náuticos sueltos en el mar (figura 3). Todo ello puede influenciar en la experiencia del turista como resultado de una mala gestión al momento de observar que el individuo avistado trata de esquivarlos o evitarlos.

\section{Conclusiones y recomendaciones}

Como producto de la revisión de la bibliografía disponible y las regulaciones internacionales, el presente manuscrito brinda un listado de ocho recomendaciones que se sugieren seguir al realizar la actividad de avistamiento de ballenas en el norte peruano. Se recomienda optar por un enfoque preventivo en el que se controlen el número de embarcaciones, el tiempo de observación, la velocidad de aproximación y la distancia entre los cetáceos y la embarcación; además de seguir y mantener un código de conducta que genere un orden entre los operadores turísticos de la zona. Estas recomendaciones permitirán un mejor control de la actividad de avistamiento de ballenas jorobadas y un manejo beneficioso para su conservación, todo lo cual hará que la actividad sea sostenible en el tiempo y se mejore la experiencia turística.

\section{Fuente de financiamiento}

Este trabajo fue autofinanciado.

\section{Potenciales conflictos de interés}

La autora declara no tener conflictos de interés.

\section{Referencias bibliográficas}

Burnham, R. E., Duffus, D. A. y Malcolm, C. D. (2021). «Towards an enhanced management of recreational whale watching: The use of ecological and behavioural data to support evidence-based management actions». Biological Conservation, 255, 109009. Disponible en: https://doi.org/10.1016/j.biocon.2021.109009

Carlson, C. (2010). «A review of whale watch guidelines and regulations around the world (version 2009)».

Castro, C., Kaufman, G. y Ponce, H. (2015). «Whale watching growth and socioeconomic development of communities in the Machalilla National Park, Ecuador». International Whaling Comission.

Chalcobsky, B. A., Crespo, E. A. y Coscarella, M. A. (2017). «Whalewatching in Patagonia: What regulation scheme should be implemented when the socio-ecological system is changing?». Marine Policy, 75, pp. 165-173. Disponible en: https://doi.org/10.1016/j. marpol.2016.11.010

Christiansen, F. y Lusseau, D. (2013). «Understanding the ecological effects of whale-watching on cetaceans». En: Whale-watching: Sustainable tourism and ecological management (pp. 177-192). Disponible en: https://doi.org/10.1017/CBO9781139018166.016
Di Clemente, J., Christiansen, F., Pirotta, E., Steckler, D., Wahlberg, M. y Pearson, H. (2018). «Effects of whale watching on the activity budgets of humpback whales, Megaptera novaeangliae (Borowski, 1781), on a feeding ground». Aquatic Conservation: Marine and Freshwater Ecosystems, 28. Disponible en: https://doi.org/10.1002/ aqc. 2909

Félix, F. (2004). «Assesment of the level of surface activity in humback whales during the breeding season». The Latin American Journal of Aquatic Mammals, 3(1), pp. 25-36. Disponible en: https://doi. org/10.5597/LAJAM00046

Garcia Cegarra, A., Gallardo, D., Pacheco, A. y Villagra, D. (2018). «Statistical dependence for detecting whale-watching effects on humpback whales». Journal of Wildlife Management, 82. Disponible en: https://doi.org/10.1002/jwmg.21602

Gleason, C. y Parsons, E. C. M. (2019). «Recent advances in whalewatching research: 2018-2019». Tourism in Marine Environments, 14. Disponible en: https://doi.org/10.3727/154427319X15645796379985

Guidino, C., Campbell, E., Alcorta, B., Gonzalez, V., Mangel, J. C., Pacheco, A. S., Silva, S. y Alfaro-Shigueto, J. (2020). «Whale watching in Northern Peru: An economic boom?». Tourism in Marine Environments, 15, pp. 1-10. Disponible en: https://doi.org/10.3727/15 $4427320 \times 15819596320544$

Guidino, C., Llapapasca, M. A., Silva, S., Alcorta, B., Pacheco, A. S. (2014). Patterns of spatial and temporal distribution of humpback whales at the southern limit of the Southeast Pacific Breeding Area». PLOS ONE, 9, e112627. Disponible en: https://doi.org/10.1371/ journal.pone.0112627

Hoyt, E. y Parsons, E. C. M. (2014). «The whale-watching industry: Historical development». En: Whale-watching: Sustainable tourism and ecological management (pp. 57-70). Disponible en: https://doi. org/10.1017/CBO9781139018166.006

Kessler, M., Harcourt, R. (2013). «Whale watching regulation compliance trends and the implications for management off Sydney, Australia». Marine Policy, 42, pp. 14-19. Disponible en: https://doi. org/10.1016/j.marpol.2013.01.016

Mackintosh, N. A. (1965). The stock of whales. Fishing News.

O'Connor, S., Campbell, R., Cortez, H., Knowles, T. (2009). Whale watching worldwide: tourism numbers, expenditures and expanding economic. International Fund for Animal Welfare.

Pacheco, A., Silva, S. y Alcorta, B. (2011). «ls it possible to go whale watching off the coast of Peru? A case study of humpback whales». Latin American Journal of Aquatic Research, 39, pp. 189-196. Disponible en: https://doi.org/10.3856/vol39-issue1-fulltext-20

Pacheco, A., Silva, S., Alcorta, B., Gubbins, S., Guidino, C., SanchezSalazar, F., Petit, A., Llapapasca, M., Balducci, N., Larrañaga, E., Zapata, M., Grados, E., Valdivia, C., Pinasco, G., Garcia Cegarra, A., Cáceres, D., Biffi, D., Silva, L., Auger, A., Jaramillo-Calle, V. (2019). «Cetacean diversity revealed from whale-watching observations in Northern Peru». Aquatic Mammals, 45, pp. 116-122. Disponible en: https://doi.org/10.1578/AM.45.1.2019.116

Pacheco, A. S., Sepúlveda, M., Corkeron, P. (2021). «Editorial: Whalewatching impacts: Science, human dimensions and management». Frontiers in Marine Science, 8, p. 1126. Disponible en: https://doi. org/10.3389/fmars.2021.737352

Rasmussen, K., Palacios, D., Calambokidis, J., Saborío, M., Dalla Rosa, L., Secchi, E., Steiger, G., Allen, J., Stone, G. (2007). «Southern hemisphere humpback whales wintering off Central America: Insights from water temperature into the longest mammalian migration». Biology Letters, 3, pp. 302-305. Disponible en: https:// doi.org/10.1098/rsbl.2007.0067 
Schuler, A. R., Piwetz, S., Di Clemente, J., Steckler, D., Mueter, F. y Pearson, H. C. (2019). «Humpback whale movements and behavior in response to whale-watching vessels in Juneau, AK». Frontiers in Marine Science, 6, p. 710. Disponible en: https://doi.org/10.3389/ fmars.2019.00710

Senigaglia, V., Christiansen, F., Bejder, L., Gendron, D., Lundquist, D., Noren, D. P., Schaffar, A., Smith, J. C., Williams, R., Martinez, E., Stockin, K. y Lusseau, D. (2016). «Meta-analyses of whalewatching impact studies: comparisons of cetacean responses to disturbance». Marine Ecology Progress Series, 542, pp. 251-263. Disponible en: https://doi.org/10.3354/meps11497

Stevick, P., Lobo, A., Allen, J., Avila, I., Capella, J., Castro, C., Chater, K., Dalla Rosa, L., Engel, M., Félix, F., Flórez-González, L., Freitas, A., Haase, B., Llanoy, M., Lodi, L., Munoz, E., Olavarria, C., Secchi, E., Scheidat, M. y Siciliano, S. (2004). «Migrations of individually identified humpack whales between the Antarctic Peninsula and South America». Journal of Cetacean Research and Management, 6, pp. 63-68.
Sprogis, K. R., Videsen, S., Madsen, P. T. (2020). «Vessel noise levels drive behavioural responses of humpback whales with implications for whale-watching». elife, 9, e56760. Disponible en: https://doi. org/10.7554/eLife. 56760

Swartzman, G., Bertrand, A., Gutiérrez, M., Bertrand, S. y Vásquez, L. (2008). «The relationship of anchovy and sardine to water masses in the Peruvian Humboldt Current System from 1983 to 2005». Progress In Oceanography, 79, pp. 228-237. Disponible en: https://doi. org/10.1016/j.pocean.2008.10.021

Villagra, D., García-Cegarra, A., Gallardo, D. I. y Pacheco, A. S. (2021). «Energetic effects of whale-watching boats on humpback whales on a breeding ground». Frontiers in Marine Science, 7, p. 1208. Disponible en: https://doi.org/10.3389/fmars.2020.600508 